\title{
Double peak rogue waves of the Sasa-Satsuma equation in a chaotic wave field
}

\author{
J. M. Soto-Crespo ${ }^{1}$, N. Devine ${ }^{2}$, N. P. Hoffmann ${ }^{3,4}$, N. Akhmediev ${ }^{2}$ \\ ${ }^{1}$ Instituto de Óptica, C.S.I.C., Serrano 121, 28006 Madrid, Spain \\ ${ }^{2}$ Optical Sciences Group, Research School of Physics and Engineering, The Australian National University, \\ Canberra ACT 0200, Australia \\ ${ }^{3}$ Dynamics Group, Hamburg University of Technology, 21073 Hamburg, Germany \\ ${ }^{4}$ Department of Mechanical Engineering, Imperial College, London SW7 2AZ, United Kingdom \\ e-mail address: j.soto@csic.es
}

\begin{abstract}
We study the properties of the chaotic wave fields generated in the frame of the Sasa-Satsuma equation, and analyze the presence of rogue waves in them. Our results also reveal the reason for the skewed profile of the exact rogue wave solutions.
\end{abstract}

OCIS codes: $190.3270,190.5940$.

Studies of rogue waves in recent years are becoming extensive [1-3]. It is an interesting object in science as researchers want to understand the physics behind the phenomenon [4]. The field also provides sufficient material for mathematicians as rogue waves can be studied using rigorous analytical tools [5-7]. Moreover, the concept of rogue waves is presently emerging in optics [8-10]. The research naturally started with the simplest nonlinear mathematical model which is the nonlinear Schrödinger equation (NLSE) [11]. This approach allowed us to find unexpected solutions even for this well known equation [12,13]. However, the NLSE has limitations related to the approximations used in its derivation.

The Sasa-Satsuma equation (SSE) is one of the known integrable extensions of the NLSE. It contains the most essential contributions often found in important physical applications, such as pulse propagation in optical fibers. Namely, it contains the terms describing third order dispersion, self-frequency shift and self-steepening in fixed proportions that make it integrable. According to the original work of Sasa and Satsuma [14], the equation can be written in the form:

$$
i \psi_{\tau}+\frac{\psi_{x x}}{2}+|\psi|^{2} \psi=i \varepsilon\left[\psi_{x x x}+3\left(|\psi|^{2}\right)_{x} \psi+6|\psi|^{2} \psi_{x}\right] .
$$

Here, the arbitrary real parameter $\varepsilon$ scales the integrable perturbations of the NLSE. When $\varepsilon=0$, Eq. (1) reduces to the standard NLSE which has only the terms describing lowest order dispersion and self-phase modulation.

Analytical solutions for rogue waves are always located on a plane wave background. The latter serves as the source from which the emerging rogue wave acquires its energy. The Sasa-Satsuma equation (1) admits the family of planewave solutions given by

$$
\psi_{0}(x, \tau)=-\frac{c}{2 \varepsilon} \exp \left[i\left(\frac{k}{2 \varepsilon} x-\frac{\omega}{8 \varepsilon^{2}} \tau\right)\right]
$$

where the amplitude parameter $c$, the wavenumber, $k$, and the frequency, $\omega$, are coupled through the dispersion relation $\omega=k^{3}+k^{2}-6 c^{2} k-2 c^{2}$. The solution has two free independent parameters, $c$ and $k$. Despite this solution looks singular with respect to the equation parameter $\varepsilon$, we can always adjust $c, k$ and $\omega$ to be of the order of $\varepsilon$. When taking the NLSE limit, $\varepsilon \rightarrow 0$, we can take $c$ and $k$ to be directly proportional to $\varepsilon$ while taking $\omega \sim \varepsilon^{2}$ to eliminate the singularity.

Equation (1) is normalized. For consistency, we consider its normalized solutions. Thus, without restricting generality, we shall deal with plane waves with the amplitude set to one. This means that we can choose the parameter $c$ to be equal to $2 \varepsilon$. More specifically, $c=1$ and $\varepsilon=0.5$. This is the choice that we keep in all numerical simulations below. This, in turn, means that the only free parameter of the plane wave solution is $k$.

One important conclusion from the expression for the plane wave is that the group velocity is different from the phase velocity. The group velocity calculated from Eq.(2) is:

$$
v_{g r}=\frac{1}{4 \varepsilon} \frac{\partial \omega}{\partial k}=\frac{3 k^{2}+2 k-6 c^{2}}{4 \varepsilon} .
$$


As we can see from here, the group velocity is negative at small $k$ and becomes positive above $k \approx 1.12$.

The exact rogue wave solution of the SSE has been presented for the first time in the work [15]. The solution is cumbersome and will not be reproduced here. Remarkably, it contains the same two free parameters $(k, c)$ as the plane wave on which it resides. The third one is $\varepsilon$, which is the parameter of the equation. An example of rogue wave solution for $k=0.25$ is shown in Figure 1(a). The solution has a double peak and is skewed to the left.

We have numerically solved the SSE, taking as initial condition the plane-wave solution (2) perturbed with white noise of small amplitude. Modulation instability seeded by this noise, creates a chaotic wave field that starts from this initial plane-wave. False color plot of a wave field generated this way is shown in Fig.1(b). The figure shows the field amplitude vs $x$ and $\tau$. Remarkably, the chaotic wave field does have rogue waves. One of them is encircled by an ellipse. It is worth noticing that the filaments that appear in this plot move with a group velocity that is approximately given by Eq.(3). Importantly enough, the exact rogue wave solution shown in Fig.1(a) has the same velocity.
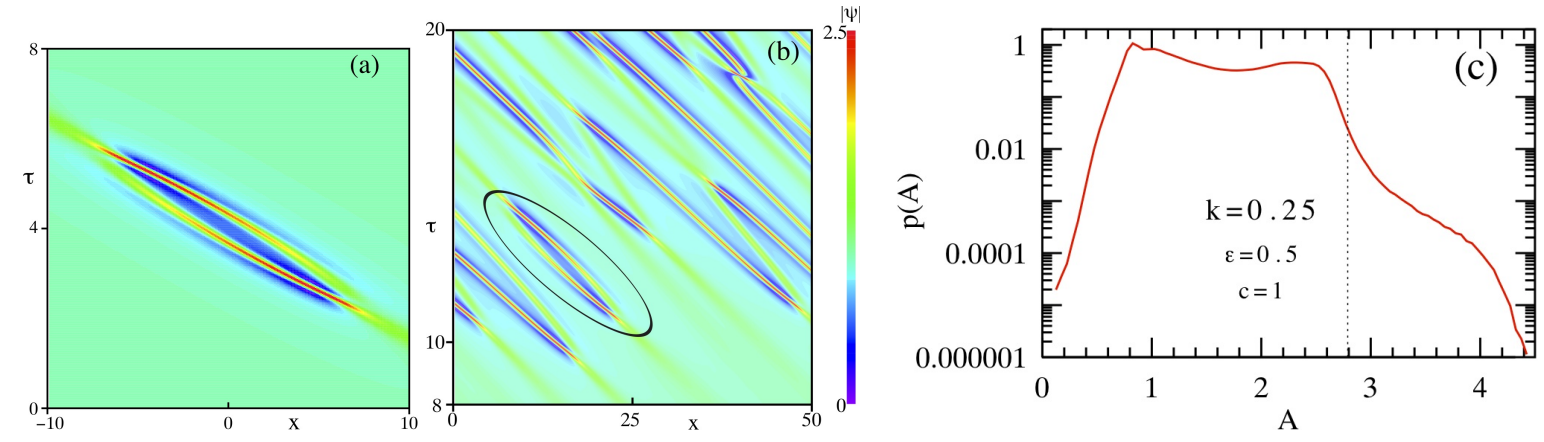

Fig. 1. (a) False color plot of a rogue wave of the SSE with parameters $c=1, \varepsilon=0.5$, and $k=0.25$. (b) Chaotic wave field created by the SSE for the same parameters. (c) Probability density function calculated using the data in (b). The peak amplitude of the rogue wave solution presented in (a) is shown in (c) by the dotted vertical line.

An example of probability density function (PDF) constructed for this chaotic wave field is shown in Fig.1(c). The red curve shows the probability, in logarithmic scale, of having a wave with a given peak amplitude. The amplitude of the exact rogue wave solution for the same value of $k$ is shown in the plot by the vertical dotted line. As we can see, a significant number of the field maxima lies above this line.

Acknowledgments: The authors acknowledge the support from the Volkswagen Stiftung. N. D. and N. A. acknowledge the support of the Australian Research Council (Discovery Project DP140100265). N. A. is a recipient of the Alexander von Humboldt Award (Germany). The work of JMSC is supported by the MINECO under contracts FIS2009- 09895 and TEC2012-37958-C02-02.

\section{References}

1. M. Onorato, S. Residori, U. Bortolozzo, A. Montina and F. T. Arecchi, Physics Reports, 528, 47 - 89 (2013).

2. N. Akhmediev and E. Pelinovsky (Editors), European Physical J., Special Topics, 185, (2010).

3. N. Akhmediev, J. M. Dudley, D. R. Solli and S. K. Turitsyn, Journal of Optics, 15, 060201 (2013).

4. C. Garrett and J. R. Gemmrich, Physics Today, No 6, p. 62, (2009).

5. Y. Ohta and J. Yang, Proceedings of Royal Society A, 468, 1716 - 1740 (2012).

6. A. Calini and C. M. Schober, Journal of Optics, 15, 105201 (1-9) (2013).

7. P. Dubard, P. Gaillard, C. Klein and V. B. Matveev, European Physical J., Spec. Topics, 185, 247 - 258 (2010).

8. D. R. Solli, C. Ropers, P. Koonath and B. Jalali, Nature, 450, 1054, (2007).

9. A. Montina, U. Bortolozzo, S. Residori and F. T. Arecchi, Physical Review Letters, 103, 173901 (2009).

10. K. Hammani, B. Kibler, C. Finot and A. Picozzi, Physics Letters A 374, 3585 - 3589 (2010).

11. N. Akhmediev, A. Ankiewicz and M. Taki, Physics Letters A, 373, 675 - 678 (2009).

12. D. J. Kedziora, A. Ankiewicz and N. Akhmediev, Physical Review E, 84, 056611 (2011).

13. D. J. Kedziora, A. Ankiewicz and N. Akhmediev, Physical Review E, 88, 013207 (2013).

14. N. Sasa and J. Satsuma, Journal of The Physical Society of Japan, 60, $409-417$ (1991).

15. U. Bandelow and N. Akhmediev, Physics Letters A, 376, 1558 - 1561 (2012). 